\title{
Intermolecular and Intramolecular Excited State Charge Transfer
}

\author{
KENNETH B. EISENTHAL
}

Department of Chemistry, Columbia University, New York, NY 10027

A primary mechanism of energy relaxation and chemical change in organic molecules in excited electronic states is charge transfer. ${ }^{1}$ The charge transfer process can be intermolecular, involving an excited molecule and a neighboring molecule, one serving as an acceptor and the other as a donor molecule, or intramolecular, involving a charge redistribution in the excited molecule which produces a very large excited state dipole moment.

In our investigations of the dynamics of these various charge transfer processes, a picosecond laser pulse was used to excite the molecules of interest. The charge transfer dynamics were monitored by a variety of techniques, including transient absorption of the excited charge transfer complex (exciplex) or ion radicals by a time delayed picosecond pulse, and fluorescence from the exciplex and from the initially excited molecule using a picosecond streak camera for detection.

\section{INTERMOLECULAR CHARGE TRANSFER-DYNAMICS OF EXCIPLEX FORMATION BETWEEN ANTHRACENE AND DIALKYLANILINE}

\subsection{Translational motions}

The increased electron affinity of photoexcited anthracene $A^{*}$ makes possible a charge transfer interaction with a ground state diethylaniline molecule in hexane at room temperature to form an exciplex. 


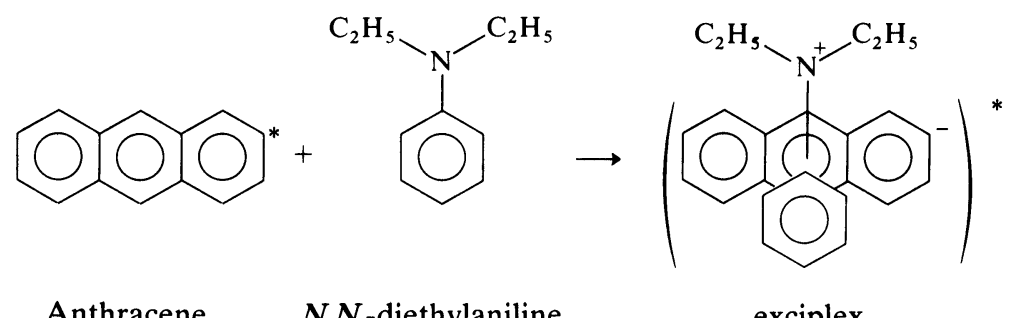

Anthracene $\quad N, N$-diethylaniline

exciplex

Information concerning the interaction distance and the reaction rate can be obtained from the formation dynamics of the exciplex. ${ }^{2}$ For an intermolecular exciplex the formation rate is controlled by the relative translational motions of the electron donor and acceptor molecules to a distance or range of distances necessary to effect a charge transfer reaction. Since the process is a rapid electron transfer process, it is likely that the reaction has a rather sharp distance dependence, i.e., the reaction has a significant probability for a very small range of intermolecular separations. Using either a diffusion model or a pair reaction model, ${ }^{3,4}$ both of which use a sharp reaction distance, one can test the adequacy of these models for describing charge transfer reactions in solution. If the measured exciplex formation rate can be described by these models, then two parameters can be extracted. One is the average radius $R$ of the reaction sphere and the other is the rate constant $k$ for reaction between donors and acceptors, separated by the distance $R$. We have found ${ }^{2}$ excellent agreement with the diffusion and Noyes reaction pair theories with experiments over a range of donor concentrations extending from $0.1 \mathrm{M}$ to $1 \mathrm{M}$. In all of these cases, the same values of $R$ and $k$ were independently found, namely $R=(8 \pm 0.5) \AA$ and $k=$ $(11 \pm 1) \times 10^{10} \mathrm{M}^{-1} \mathrm{~s}^{-1}$. At $3 \mathrm{M}, 6 \mathrm{M}$ and neat donor, the reaction was so rapid that we could only estimate that the reaction rate of $A^{*}$ with a neighboring diethylaniline donor was greater than $10^{11} \mathrm{~s}^{-1}$.

\subsection{Rotational motion of the exciplex}

By using a polarized light pulse to excite the anthracene molecule and probing the exciplex absorption with polarized light, it is possible to obtain the rotational relaxation time of the exciplex. Although the theoretical treatment ${ }^{2}$ of the orientational relaxation includes the 
rotational motions of $A^{*}$ prior to exciplex formation the experiment was carried out in neat diethylaniline to minimize the time that $A^{*}$ is unreacted and thereby enhance the contributions of $\left(A^{-}-D^{+}\right)^{*}$ to the overall rotational relaxation process. The results ${ }^{2}$ indicate that the orientational relaxtion time of the exciplex is 60 ps which is in reasonable agreement with $\tau_{0 r}$ obtained from the Debye relation $\tau_{0 r}=\eta V / k T$ using an estimated volume for a sandwich structured exciplex.

In addition, the transition moment

$$
\left(A^{-}-D^{+}\right)^{*} \stackrel{694 \mathrm{~nm}}{\longrightarrow}\left(A^{-}-D^{+}\right) * *
$$

is found to be perpendicular to the $A \rightarrow A^{*}$ short axis transition at $347 \mathrm{~nm}$. Since the exciplex transition at $694 \mathrm{~nm}$ is primarily due to absorption by the $\boldsymbol{A}^{-}$part of the complex, the exciplex transition at $694 \mathrm{~nm}$ is parallel to the long axis of anthracene.

\subsection{Geometric requirements}

To investigate the geometric requirements for exciplex formation, one approach is to restrict the relative orientation of the electron donor and acceptor molecules by connecting them with a number of methylene groups. The effect of this restriction on the relative orientation of the donor and acceptor, while still maintaining their close proximity $(<7 \AA)$, can then be studied. The molecule that we have studied is

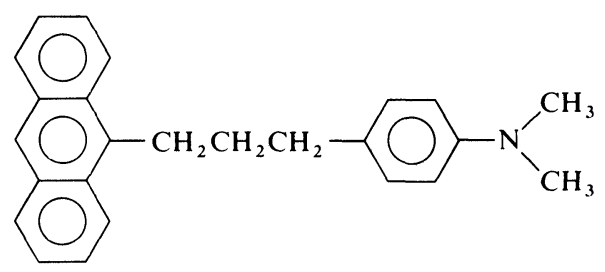

Upon excitation of the anthracene part, this model molecule should form an exciplex as rapidly as the free anthracene and DMA system, as described earlier, if there are no significant orientational requirements for CT interaction. On the other hand, if the time course for exciplex formation is different for the two cases, then molecular motions to achieve a favorable geometry must be involved and can be directly followed by monitoring the formation of the exciplex. 
The absorption and fluorescence spectra of the model molecule are the same as that of the free molecules, indicating that there are no significant ground state interactions between the two moieties. A picosecond laser pulse at $351 \mathrm{~nm}^{5,6}$ was used to excite the anthracene moiety in the model compound. The decay of the anthracene fluorescence and the rise of the exciplex fluorescence were then monitored by a streak camera. In Figures 1 and 2 we show the decays of anthracene fluorescence monitored at $410 \mathrm{~nm}$ and the rises of exciplex fluorescence monitored at $520 \mathrm{~nm}$ in four nonpolar solvents (isopentane, hexane, decane, and tetradecane). The fluorescence curve for exciplex formation consists of a very rapid initial rise (within the laser pulse width), which is due to the tail of the overlapping anthracene emission, followed by a much slower rise. By subtracting the contribution of the anthracene emission, a true exciplex formation curve can be obtained. Several conclusions can be drawn from these data.

1) If there were no geometric requirements for exciplex formation, then we know from the earlier results that a rise time of $\sim 10 \mathrm{ps}$

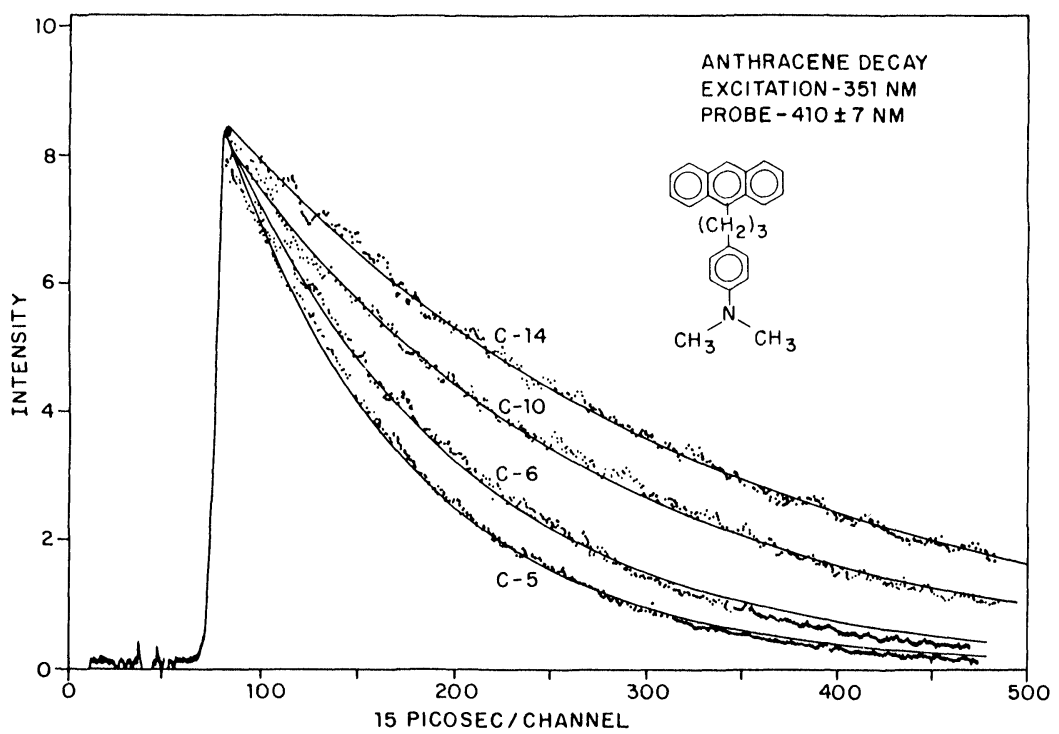

FIGURE 1 The fluorescence decay curves of anthracene moiety in isopentane $\left(\mathrm{C}_{5}\right)$, hexane $\left(C_{6}\right)$, decane $\left(C_{10}\right)$, and tetradecane $\left(C_{14}\right)$. The points are experimental, the solid curves are theoretical. 


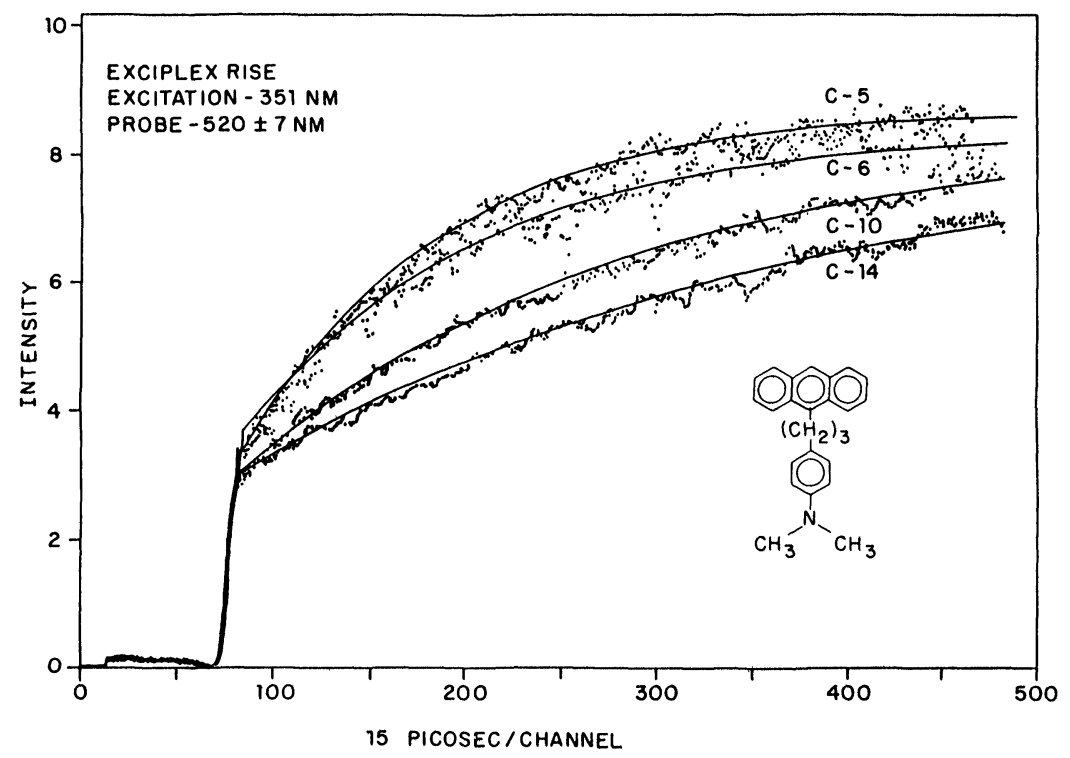

FIGURE 2 The fluorescence rise curves of intramolecular exciplex in isopentane $\left(C_{5}\right)$, hexane $\left(C_{6}\right)$, decane $\left(C_{10}\right)$, and tetradecane $\left(C_{14}\right)$. The points are experimental, the solid curves are theoretical.

should be obtained. The much slower formation rate and the strong dependence on solvent viscosity which we find, indicate that rotational motions are required to bring the two moieties into a favorable conformation to form the exciplex. The time dependence of this motion also provides direct information about the end-to-end relaxation dynamics of this short chain molecule. ${ }^{6}$

2) The anthracene decay times and the exciplex formation times are found to be the same in all the solvents studied, thus indicating that there is no long-lived non-fluorescent intermediate state present before the formation of the fluorescent exciplex.

Although these results indicate strong geometrical requirements for exciplex formation in the anthracene- $\left(\mathrm{CH}_{2}\right)_{3}$-dimethylaniline molecule different behavior is exhibited by other exciplex systems. Studies ${ }^{7,8}$ of the naphthalene- $\left(\mathrm{CH}_{2}\right)_{n}-\mathrm{N}\left(\mathrm{CH}_{3}\right)_{2}$ system in methylcyclohexane and isopentane solutions, found no strong geometrical 
preference for exciplex formation. This conclusion was based on the observation that exciplexes were formed when $n=2,3$, or 4 . In the study $^{9}$ of intramolecular exciplex formation for the molecule $\mathrm{N}$ carbozolyl- $\left(\mathrm{CH}_{2}\right)_{n}$-tetrachloropthalimide in cyclohexane solution, it was concluded that there are no geometric requirements for exciplex formation. This was based on the following: (1) the exciplex can be formed for chains in which $n=2,3,4$, and 7, and (2) the formation rates are extremely fast (shorter than 200 ps) and virtually independent of solvent viscosity. These different observations can be rationalized by assuming that the degree of geometric constraint imposed on exciplex formation becomes less important as the driving force of the reaction increases. A measure of the driving force is the free energy change of the reaction which can be expressed in terms of the experimentally measured energy separation between the locally excited singlet and exciplex states. ${ }^{1}$ These energy differences are about 1.0, 0.5 , and $0.13 \mathrm{eV}$ for $\mathrm{N}$-carbazolyl- $\left(\mathrm{CH}_{2}\right)_{n}$-tetrachlorophthalimide, naphthalene- $\left(\mathrm{CH}_{2}\right)_{n}-\mathrm{N}\left(\mathrm{CH}_{3}\right)_{2}$, and anthracene- $\left(\mathrm{CH}_{2}\right)_{n}$-dimethylaniline, respectively, which is consistent with the observed trend of geometric constraint on exciplex formation. This view is further supported by the observation ${ }^{10}$ than an exciplex $A-\left(\mathrm{CH}_{2}\right)_{n}-D$ will form in polar solvents but not in nonpolar solvents when $n=1$ or 2 . This result can be understood by considering the decrease in the free energy change (i.e., $\Delta G$ becomes more negative) as the solvent polarity increases. The resulting increased driving force reduces the importance of the geometric constraint on exciplex formation. As the solvent dielectric constant increases further, there will be progressively less geometric constraint on charge transfer. Eventually solvent stabilization will make an ion-pair state accessible from the exciplex state as well as from the locally excited state. This latter pathway opens up a direct electron-transfer channel for energy relaxation and will be discussed in the following section.

The effect of solvent polarity The CT interactions, though determined by the intrinsic properties of the donor and acceptor pair, are greatly modified by environmental effects. Due to the charge separation associated with the $\mathrm{CT}$ interaction the solvent polarity can play an important role. To examine the interplay between geometric and solvent dielectric effects, we have studied anthracene- $\left(\mathrm{CH}_{2}\right)_{3}$ dimethylaniline in the strongly polar $(\varepsilon=37)$ solvent acetonitrile. 
The anthracene moiety was excited at $351 \mathrm{~nm}$ and the weak exciplex emission monitored in the 500-600 nm region. In marked contrast to the results in nonpolar solvents, where the exciplex is formed in a few nanoseconds and lives for more than one hundred nanoseconds, we have found ${ }^{11}$ in acetonitrile that it is formed in a time shorter than we can resolve $(<2 \mathrm{ps})$ and decays in $580 \pm 30 \mathrm{ps}$, as shown in Figure 3. Although the exciplex is formed "instantaneously," we find that the excited anthracene moiety, which is monitored at $411 \mathrm{~nm}$, has a lifetime of $7 \pm 1 \mathrm{ps}$, Figure 4 . Since the CT rise is faster than the anthracene decay we conclude that these observed emissions correspond to independent processes.

The very fast CT rise suggests that only molecules in a favorable ground state conformation at the time of excitation lead to the exciplex. For those molecules in unfavorable extended conformations a new nonradiative decay channel is responsible for the observed $7 \mathrm{ps}$ lifetime of the excited anthracene moiety. We believe this new ultrafast decay channel is direct $e^{-}$transfer to form a nonfluorescent ion-pair which is strongly stabilized by the polar solvent. The internal rotational motions necessary for the formation of the exciplex are

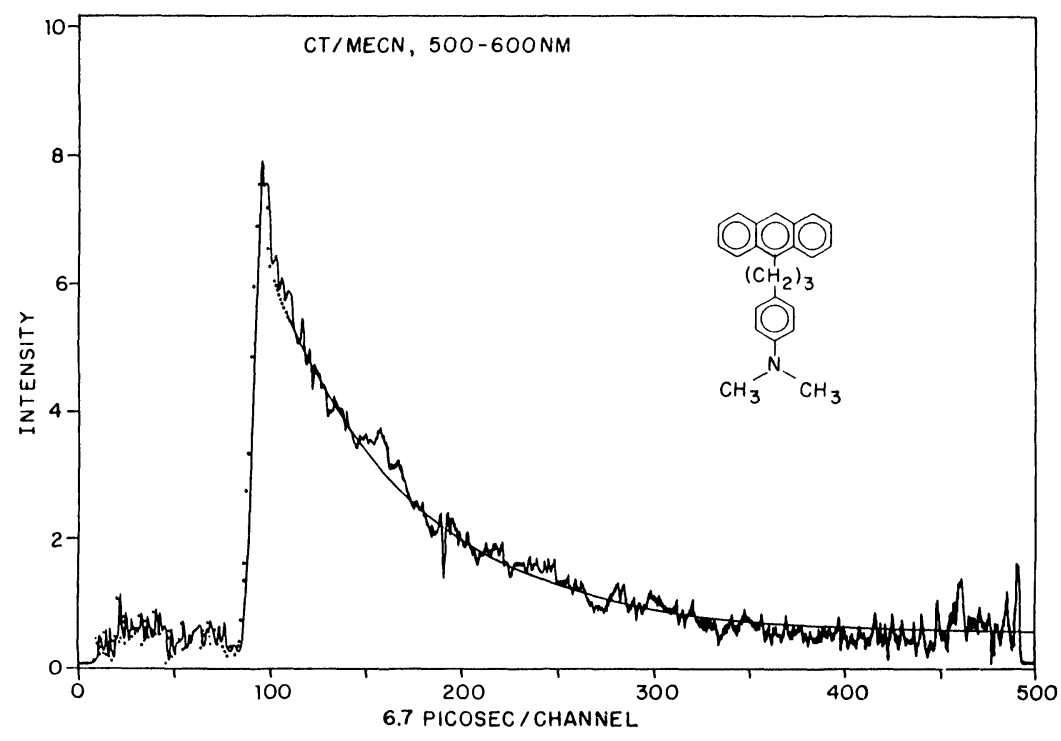

FIGURE 3 The fluorescence decay curve of the $A-\left(\mathrm{CH}_{2}\right)_{3}-D$ exciplex in acetonitrile. 


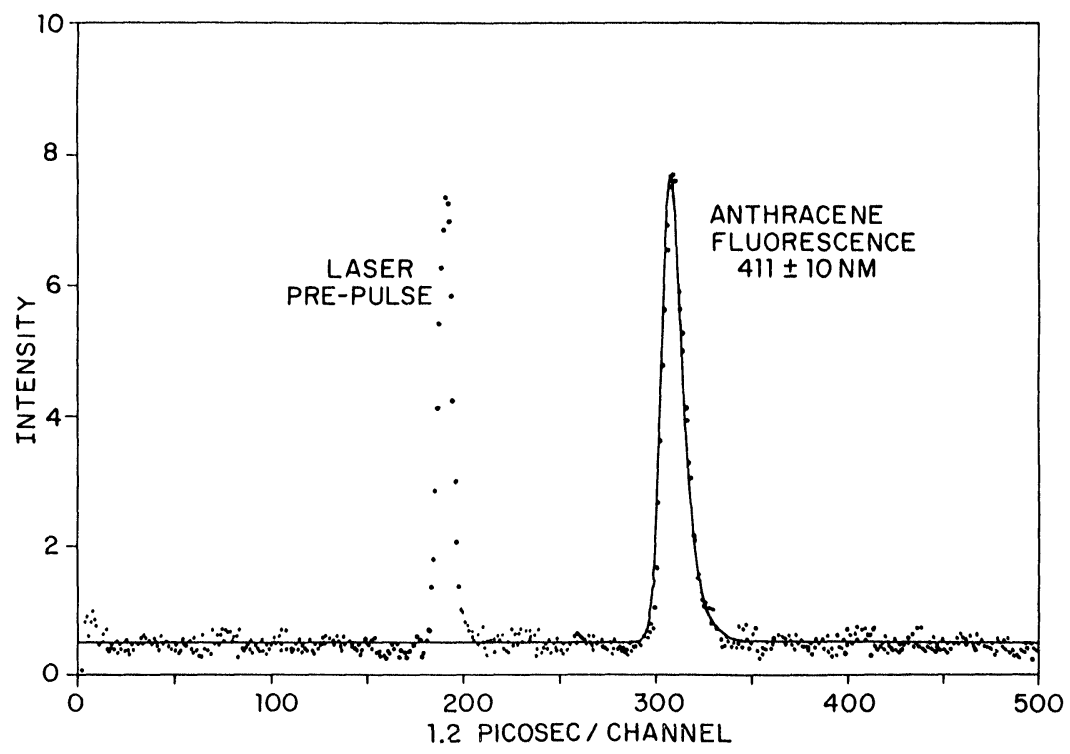

FIGURE 4 The fluorescence decay curve of anthracene moiety in acetonitrile.

too slow to compete in this case. This is confirmed by the direct observation of ion-pair formation ( $\leqslant 10 \mathrm{ps)} \mathrm{observed} \mathrm{by} \mathrm{its} \mathrm{charac-}$ teristic absorption at $694 \mathrm{~nm}$, as shown in Figure 5.

The appearance of this new channel for energy dissipation in polar solvents is a result of solvent stabilization of the ion pair. On the other hand, in the non-polar solvents, e.g., 2-methylbutane, it is probable that the energy of $A^{-}-\left(\mathrm{CH}_{2}\right)_{3}-D^{+}$is higher than that of $A^{*}{ }_{-}\left(\mathrm{CH}_{2}\right)_{3}-D$ in extended conformations. The following scheme summarizes the results in very polar media:

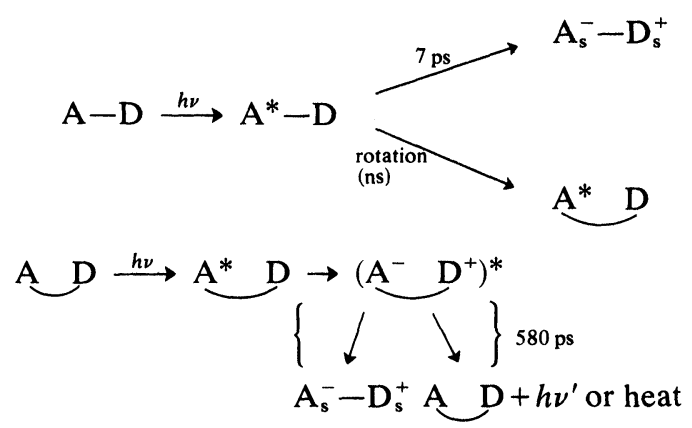




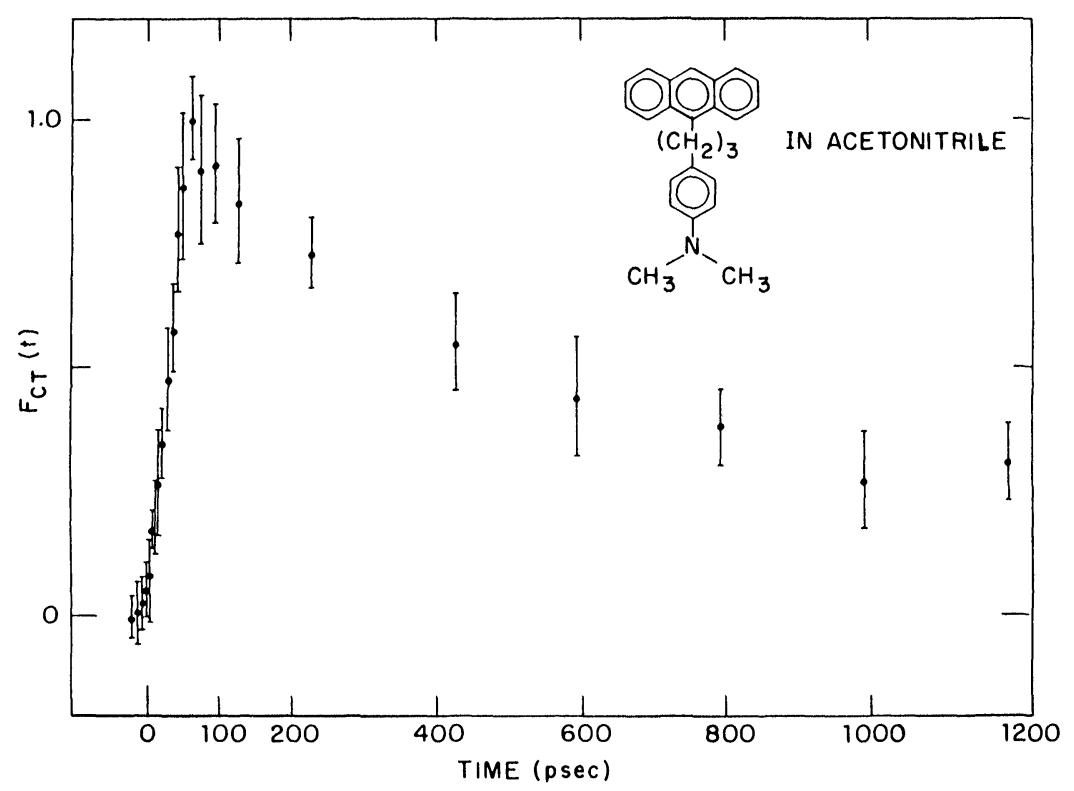

FIGURE 5 The time-dependent absorption curve of ion-pair in acetonitrile.

where $A \quad D$ represents the sandwich conformation, $A-D$, an extended conformation $A_{s}^{-}-D_{s}^{+}$the solvent stabilized extended ion pair, and $\left(\begin{array}{ll}A & D^{+}\end{array}\right)^{*}$ the excited CT complex.

Combining the present findings in acetonitrile with results in nonpolar solvents, we can make some general remarks on the chargetransfer dynamics of $A-\left(\mathrm{CH}_{2}\right)_{3}-D$ in liquids. The key to understanding the behavior of these charge-transfer systems is the strong dependence of the energies of the excited charge-transfer complex and ion-pair states on both conformation and solvent polarity. The shifts in the energies of these states relative to each other and to the energy of the locally excited state (which is not very solvent or conformation sensitive) determine the decay channels. Our experimental results in acetonitrile and 2-methylbutane, presented in Figure 6, represent the extreme cases. In the non-polar solvent the lowest-energy state for the extended conformation is the locally excited one, $A^{*}-D$. In the folded conformation the excited CT state $\left(A^{-} D^{+}\right)^{*}$ becomes more stable than $A^{*} D$. Thus to effect charge transfer the extended 


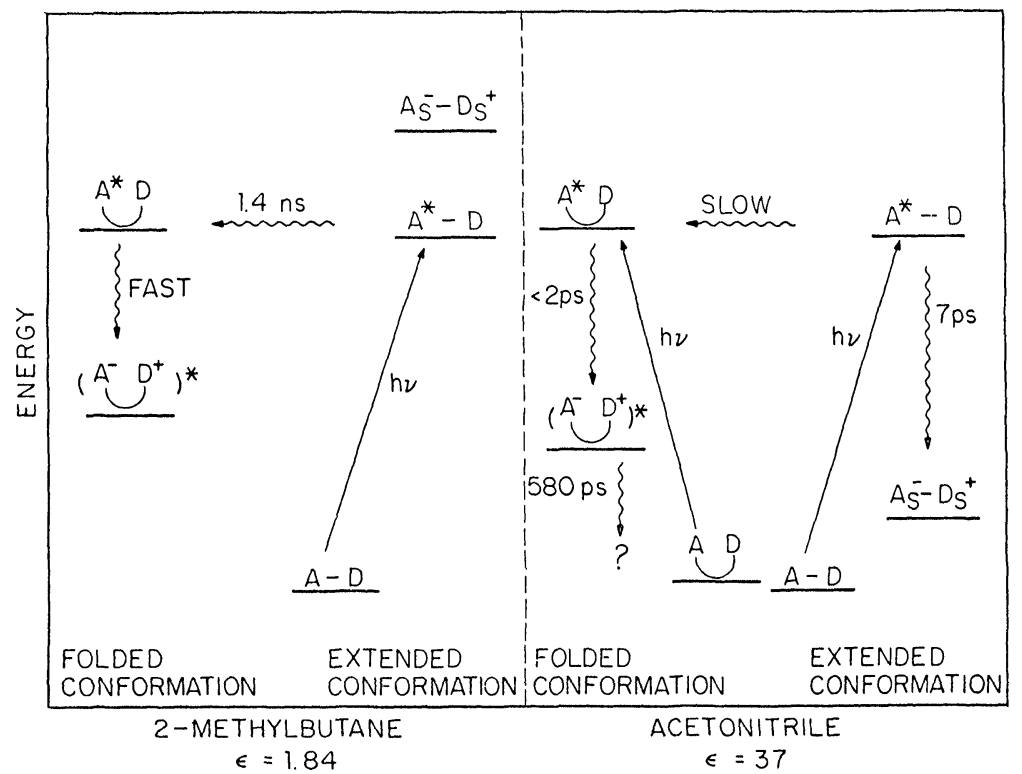

FIGURE 6 Energy levels and dynamics of anthracene- $\left(\mathrm{CH}_{2}\right)_{3}-\mathrm{N}, \mathrm{N}$-dimethylaniline in both a low- and high-polarity solvent. Relative energies are qualitative.

molecules must assume the folded conformation. In acetonitrile, on the other hand, the lowest-energy state is the solvated extended ion-pair conformation, $A_{s}^{-}-D_{s}^{+}$. A direct electron transfer to go from $A^{*}-D$ to this state is therefore energetically feasible and extremely fast $(7 \mathrm{ps})$. For the folded conformation, $\left(A^{-} D^{+}\right)^{*}$ is at a lower energy than $A^{*} \quad D$ and thus exciplex formation becomes the most rapid energy decay route. We find this process to be faster than we can resolve $(<2 \mathrm{ps})$. Since the solvated extended ion pair, $A_{s}^{-}-D_{s}^{+}$, is lower in energy than the folded excited CT complex, $\left(A^{-} D^{+}\right)^{*}$, one channel through which this latter species can relax is by unfolding and undergoing solvent stabilization. If we now consider liquids of intermediate polarity, the formation rate of the ion pair should slow down sufficiently (compared with the very rapid rate in acetonitrile) to permit the chain motions necessary for development of the folded excited CT complex from the extended form. Therefore, both exciplex and direct ion-pair formation should be observed. 


\section{DYNAMICS OF INTRAMOLECULAR CHARGE TRANSFER IN $p$-(DIMETHYLAMINO) BENZONITRILE (DMABN)}

In the previous examples of charge transfer, the $A$ and $D$ moieties were, prior to reaction, distinct molecules, whether free or hooked together by the inert $\mathrm{CH}_{2}$ groups. For the case considered now, the $A$ and $D$ moieties are electronically part of the same molecule and the CT process intramolecular in nature. The charge transfer process in DMABN and related molecules, often manifested by a dual fluorescence in polar media, has been a subject of considerable investigation. ${ }^{12-25}$ In nonpolar solvents, excitation of the DMABN molecule results in a single fluorescence band centered around $340 \mathrm{~nm}$. However, in polar solvents, a new red shifted fluorescence appears, which is associated with a large dipole moment of $\sim 16 \mathrm{D} .^{21}$ To explain the origin of this new emission band, a number of mechanisms have been proposed. ${ }^{12-23}$ The results accumulated over the past several years indicate that both the bond twisting (between the amino group and the benzene ring) and solvation play important roles in causing the charge redistribution. ${ }^{21-23}$

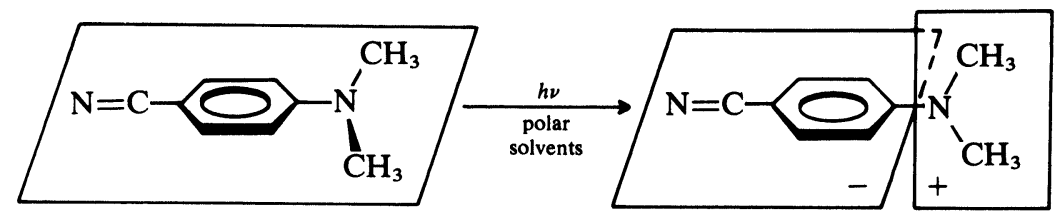

In spite of the numerous studies on the subject, the relationship between the two fluorescence bands was, surprisingly, not fully understood until recently. ${ }^{26}$ By using picosecond fluorescence spectroscopy, we have sought to determine the relationship between these emissions and most importantly, the role of the solvent in the charge transfer process.

\subsection{Neat alcohol solutions}

DMABN in neat propanol and in neat butanol were excited with a single $264 \mathrm{~nm}$ ps laser pulse. The "normal" short wavelength emission and the CT emission were then monitored at 350 and $470 \mathrm{~nm}$, respectively, by a streak camera. In both propanol ${ }^{26}$ and butanol ${ }^{27}$ 
solutions, the short wavelength fluorescence decay can be clearly resolved into two exponential components. The fast component decays with a lifetime of $19 \pm 3$ ps in propanol solution and $30 \pm 5$ ps in butanol solution, which are equal to the risetimes of the CT fluorescence in the corresponding liquids. The slow decay component at $350 \mathrm{~nm}$ has the same lifetime as the decay of the CT emission, being $2.3 \pm 0.2 \mathrm{~ns}$ in propanol and $2.2 \pm 0.2 \mathrm{~ns}$ in butanol.

These data clearly demonstrate that in pure alcohol solutions an equilibrium between the two emitting species is rapidly established after excitation. This equilibrium can be described by

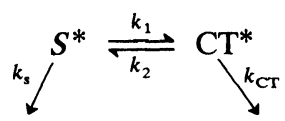

where $S^{*}$ and $\mathrm{CT}^{*}$ represent the states responsible for the short wavelength and long wavelength emissions respectively, $k_{s}$ and $k_{\mathrm{CT}}$ represent the radiative and nonradiative decay rates for $S^{*}$ and $\mathrm{CT}^{*}$, respectively, exclusive of the interconversion rates. As shown in Figure 7, an excellent fit was obtained between the experimental data

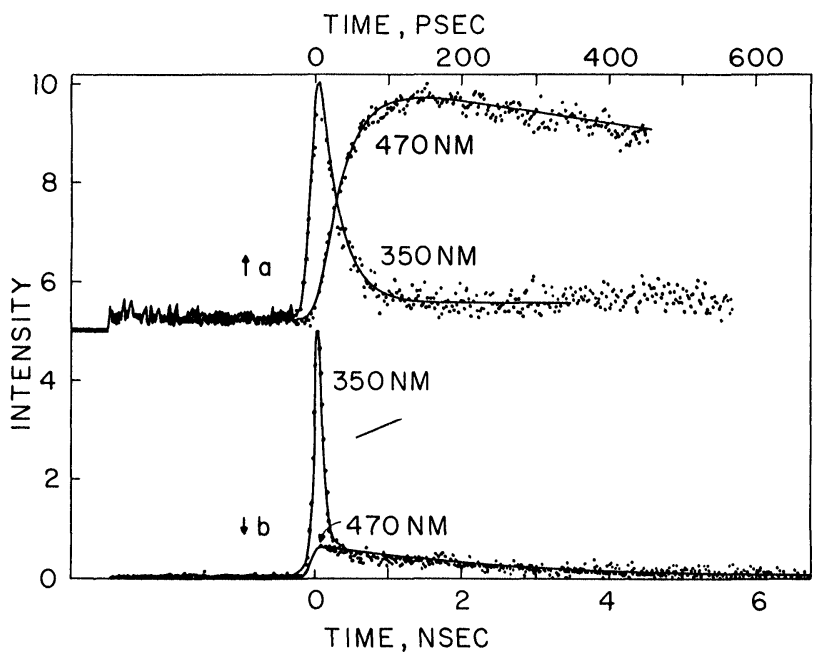

FIGURE 7 Flourescence decay curve monitored at $350 \mathrm{~nm}$ and rise curve monitored at $470 \mathrm{~nm}$ of $2.5 \times 10^{-4} \mathrm{M}$ DMABN/butanol solution excited at $264 \mathrm{~nm}$. Arrows indicate the associated time scales. (a) On the fast streak speed $0.006 \mathrm{~ns} / \mathrm{mm}$; (b) on the slow streak speed $0.6 \mathrm{~ns} / \mathrm{mm}$. 
and the theoretical curves calculated from the coupled differential equations based on the above equilibrium relationship.

The picosecond kinetic measurements in establishing both a viscosity dependence and the presence of two species supports the twisted internal charge transfer (TICT) mechanism. This mechanism suggests that the intramolecular charge transfer of DMABN molecule is induced by rotation around the $\mathrm{C}-\mathrm{N}$ bond between the amino group and the benzene ring. The short wavelength emitting species, identified with the planar form of DMABN, and the long wavelength emitting species, the perpendicular form, are in equilibrium.

\subsection{Butanol/hexadecane solutions}

To study the role of solvent molecules in the intramolecular CT process of excited DMABN molecule and hopefully to gain a better understanding of the coupling between the twisting motion and solvent relaxation, the dynamics of the $\mathrm{CT}$ transfer process in mixed solvents were investigated. ${ }^{27}$ The basic idea is to control the local number density of the polar solvent molecules without greatly altering the viscosity of the solution. The effect of the change in local dipole density on the dynamics and yield of the CT process could then yield information on the role of the polar solvent molecules. A convenient way to adjust the local dipole density is by diluting the polar solvent molecules with nonpolar molecules. We have chosen to study the mixed solvent system butanol/hexadecane because of their similar viscosities 3.0 and $3.3 \mathrm{cp}$ at $20^{\circ} \mathrm{C}$, respectively.

Picosecond dynamics The evolution of the photoexcited DMABN molecule from the initial planar structure to the final perpendicular structure was followed by monitoring both the singlet fluorescence decay at $350 \mathrm{~nm}$ and the CT fluorescence rise at $470 \mathrm{~nm}$ as the butanol solution is successively diluted by the non-polar hexadecane molecules. In Figure 8, the fluorescence rise curves of the CT state at several butanol concentrations are shown. In pure butanol solution, the rise curve can be fitted by a single exponential with formation time of $30 \mathrm{ps}$. When the solution is diluted by hexadecane, two components appear in the rise curves. The magnitude of the fast component decreases as the butanol concentration decreases; but its

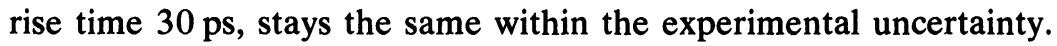




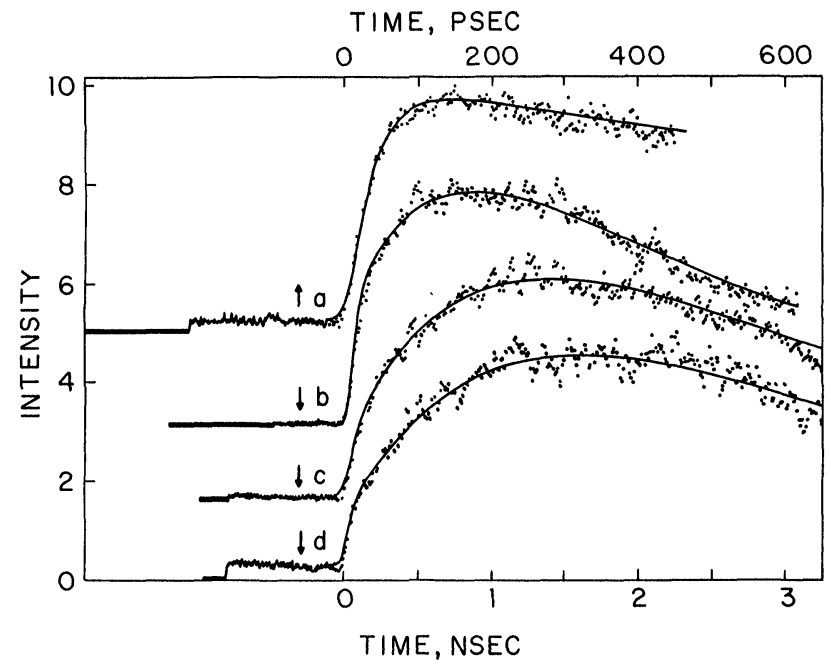

FIGURE 8 Fluorescence rise curves monitored at $470 \mathrm{~nm}$ of $2.5 \times 10^{-4} \mathrm{M}$ DMABN in mixed butanol/hexadecane solutions excited at $264 \mathrm{~nm}$. Arrows indicate the associated timescales. (a) pure butanol; (b) $[$ butanol $]=1.82 \mathrm{M}$; (d) [butanol] $=0.42 \mathrm{M}$.

On the other hand, the rate of the slow formation component increases linearly with the butanol concentration. Since the excited DMABN concentration is much less than the butanol concentration, the observed reaction can be treated as a pseudo-first-order process. The plot of this pseudo-first-order constant vs. butanol concentration yields a straight line (Figure 9). The pseudo-first-order rate constant, being equal to the product of the bimolecular rate constant and the butanol concentration, the slope of the line $\left(9.7 \pm 1.5 \times 10^{8} \mathrm{M}^{-1} \mathrm{~s}^{-1}\right)$ yields the bimolecular rate constant for the reaction of excited DMABN with butanol. It should be noted that this rate constant is smaller than that for a diffusion controlled reaction and thus its value gives the intrinsic rate constant between the reacting moieties.

The decay kinetics of the "normal" blue emission at $350 \mathrm{~nm}$ has also been analyzed. We find that results obtained from the decay kinetics at $350 \mathrm{~nm}$ are the same as the formation kinetics obtained at $470 \mathrm{~nm}$, i.e., a fast decay component of $30 \mathrm{ps,} \mathrm{independent} \mathrm{of}$ butanol concentration, followed by a slower component whose rate and magnitude depends on the butanol concentration. 


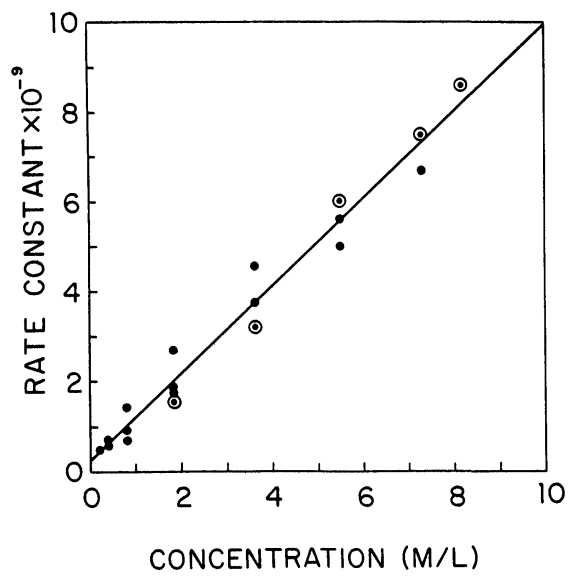

FIGURE 9 The plot of the first order rate constant of the slow component as a function of the butanol concentration. Solid circles, $\boldsymbol{\Theta}$, are from the rise curves. Open circles, $\odot$, are from the decay curves.

To interpret these interesting results, we focus on three key observations, namely, (1) the appearance of a fast and a slow kinetic process upon the dilution of butanol solutions, (2) the dynamics of the fast process is independent of the dilution while the rate of the slow process shows a linear dependence on the butanol concentration, and (3) the relative importance of the two processes is affected by the dilutions, the fast process dominates at high butanol concentration.

The origin of the fast process (i.e., the 30 ps rise observed for all butanol concentrations), we believe, is due to the excitation of the complexes already formed in the ground state between DMABN and butanol molecules. This $30 \mathrm{ps}$ rise is faster and larger than can be accounted for by a diffusion controlled process for the lower butanol concentrations. The formation of the ground state complexes is really not surprising in light of the large dipole moment $7 \mathrm{D}$ of the ground state DMABN molecule. ${ }^{17}$ According to our interpretation, the measured $30 \mathrm{ps}$ risetime for the fast process is then mainly due to the intramolecular bond twisting motion along the $\mathrm{C}-\mathrm{N}$ bond of the excited DMABN-butanol complex. This intramolecular twisting motion can be impeded by the frictional force of the solvent molecules, thus yielding a viscosity dependence. 
As the rapid rise component $(30 \mathrm{ps})$ reflects the population of ground state complexes, the slow component of the rise reflects those excited DMABN molecules which either do not have neighboring butanol molecules or do not have the proper arrangement of butanol molecules for reaction to occur. Even at the higher alcohol concentrations, for which there are several alcohol molecules, on the average, surrounding a ground state DMABN molecule, a slow component is observed. It is not until the appropriate configuration is achieved, as given by the slower time component, that the rapid $30 \mathrm{ps}$ reaction can occur. The key feature in the stabilization of the twisted intramolecular charge transfer structure appears to be the prior formation of a complex between excited DMABN and some butanol molecule or molecules.

With regard to the observed linear dependence of the slower kinetic compound on butanol concentration, we note that this result does not necessarily imply that the reaction of DMABN* is with one butanol molecule or for that matter, with any particular butanol oligomer, albeit dimer, trimer, etc. The linearity of the reaction rate is obtained by plotting the total butanol concentration as given in Figure 9, without consideration of the aggregation of the alcohol, which we do not know in any case. For the aforementioned description of the reaction of DMABN* with one butanol moiety to be correct, would require the concentration of the reacting butanol moiety (monomer, dimer, etc.) to scale linearly with the total butanol concentration over a considerable concentration range. This linear scaling is not known to be the case, nor anticipated, at high alcohol concentrations. A description that is compatible with the observed linearity is one in which the reaction of the excited DMABN molecule with butanol, producing a complex is insensitive to whether the butanol molecule is a monomer or part of some molecular aggregate (dimer, etc.). The details of the ground state complex, i.e., whether it is a $1: 1$ or $1: n$ complex, does not seem to be crucial to the dynamics of TICT formation. Support for this is obtained by noting that the same $30 \mathrm{ps}$ risetime is found for all of the butanol solutions, $0.19-11 \mathrm{M}$ (neat butanol), despite our expectation that the relative populations of butanol monomer, dimers, etc., would change significantly over this wide concentration range.

Our proposed mechanism can be briefly summarized in the figure shown below, noting that the single butanol entity shown in the figure can be monomeric or part of some higher aggregate. 


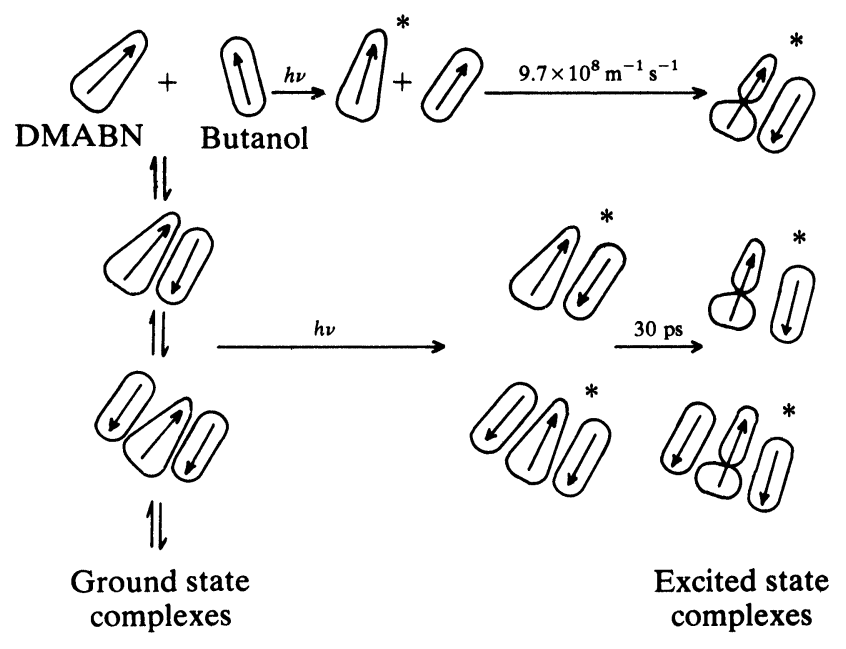

Following the stabilization of the twisted charge transfer state by the short-range interaction with neighboring polar molecules, a further stabilization of the twisted structure is achieved by the long-range polarization interaction with the solvent. This is reflected in the continuing shift of the charge transfer emission maximum to the red as the butanol concentration is increased. In summary, we note that the observed kinetics, absorption and dual emission spectra in pure and mixed solvents can be explained by the mechanism outlined above.

\section{References}

1. N. Mataga and M. Ottolenghi, in: Molecular Association, Vol. 2, ed. R. Forster (Academic Press, New York, 1979) p. 1.

2. T. J. Chuang and K. B. Eisenthal, J. Chem. Phys. 62, 2213 (1975).

3. R. M. Noyes, Progr. React. Kinetics 1, 129 (1961).

4. A. H. Alwattar, M. D. Lumb and J. B. Birks, in: Organic Molecular Photophysics, Vol. 1, ed. J. B. Birks (John Wiley \& Sons, New York, 1973) Chapter 8.

5. Y. Wang, M. K. Crawford and K. B. Eisenthal, J. Phys. Chem. 84, 2696 (1980).

6. Y. Wang, M. K. Crawford and K. B. Eisenthal, JACS 104, 5974 (1982).

7. E. A. Chandross and H. T. Thomas, Chem. Phys. Lett. 9, 393 (1971).

8. R. S. Davidson and V. R. Tretheway, J. Chem. Soc., Chem. Commun. 827 (1976).

9. J. H. Borkent, A. J. W. DeJong, J. W. Verhoeven and Th. J. DeBoer, Chem. Phys. Lett. 57, 530 (1978).

10. R. Ide, Y. Sakata and S. Misumi, J. Chem. Soc., Chem. Commun. 1009 (1972).

11. M. K. Crawford, Y. Wang and K. B. Eisenthal, Chem. Phys. Lett. 79, 529 (1981).

12. E. Lippert, W. Lüder and H. Boos, Advan. Mol. Spectrosc. 443 (1962). 
13. O. S. Khalil, J. L. Meeks and S. P. McGlynn, Chem. Phys. Lett. 39, 457 (1976).

14. H. Dodiuk and E. M. Kosower, Chem. Phys Lett. 34, 253 (1975).

15. E. A. Chandross, in: The Exciplex, eds. M. Gordon and W. R. Ware, (Academic Press, New York, 1975) p. 187.

16. K. Rotkiewicz, K. H. Grellmann and Z. R. Grabowski, Chem. Phys. Lett. 19, 315 (1973).

17. K. Rotkiewicz and W. Rubaszenska, Chem. Phys. Lett. 70, 444 (1980).

18. K. Rotkiewicz, Z. R. Grabowski, A. Krowczynski and W. Kühnle, J. Lumin. 12/13, 877 (1976).

19. J. Lipinski, H. Chojnacki, Z. R. Grabowski and K. Rotkiewicz, Chem. Phys. Lett. 58, 379 (1978).

20. E. Kirko-Kaminska, K. Rotkiewicz and A. Grabowska, Chem. Phys. Lett. 58, 379 (1978).

21. Z. R. Grabowski, K. Rotkiewicz, A. Siemiarczuk, D. J. Cowley and W. Baumann, Jouv. J. Chim. 3, 443 (1979).

22. W. Rettig, G. Wermuth and E. Lippert, Ber. Bunsenges. Phys. Chem. 83, 692 (1979).

23. W. Rettig and V. Banačić-Koutecký, Chem. Phys. Lett. 62, 115 (1979).

24. N. Nakashima, H. Inoue, N. Mataga and C. Yamanaka, Bull. Chem. Soc. Jpn. 46, 2288 (1973).

25. W. S. Struve, P. M. Rentzepis and J. Jortner, J. Chem. Phys. 59, 5014 (1973).

26. Y. Wang, M. McAuliffe, F. Novak and K. B. Eisenthal, J. Phys. Chem. 85, 3736 (1981).

27. Y. Wang and K. B. Eisenthal, J. Chem. Phys. 77, 6076 (1982). 